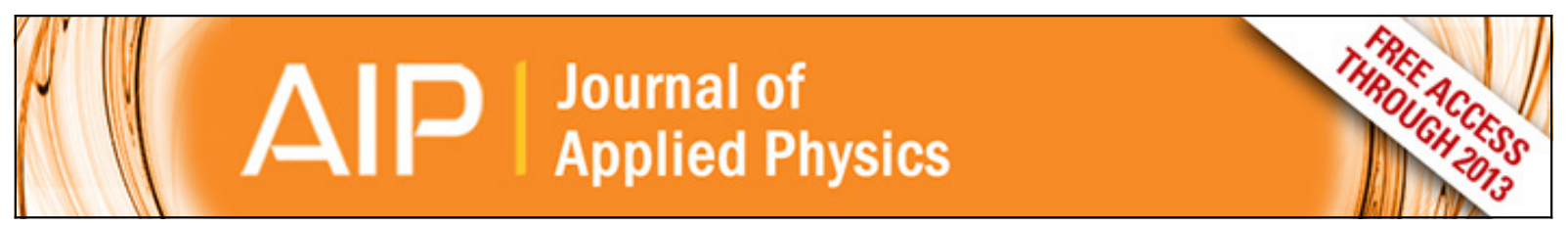

Analysis of lateral transport through the inversion layer in amorphous silicon/crystalline silicon heterojunction solar cells

Miha Filipi, Zachary C. Holman, Franc Smole, Stefaan De Wolf, Christophe Ballif, and Marko Topi

Citation: Journal of Applied Physics 114, 074504 (2013); doi: 10.1063/1.4818709

View online: http://dx.doi.org/10.1063/1.4818709

View Table of Contents: http://scitation.aip.org/content/aip/journal/jap/114/7?ver=pdfcov

Published by the AIP Publishing

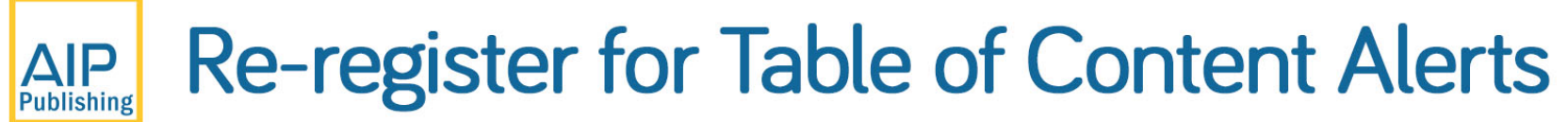




\title{
Analysis of lateral transport through the inversion layer in amorphous silicon/crystalline silicon heterojunction solar cells
}

\author{
Miha Filipič, ${ }^{1, a)}$ Zachary C. Holman, ${ }^{2}$ Franc Smole, ${ }^{1}$ Stefaan De Wolf, ${ }^{2}$ Christophe Ballif, ${ }^{2}$ \\ and Marko Topič ${ }^{1}$ \\ ${ }^{1}$ Faculty of Electrical Engineering, University of Ljubljana, Tržaška 25, SI-1000 Ljubljana, Slovenia \\ ${ }^{2}$ Ecole Polytechnique Fédérale de Lausanne (EPFL), Institute of Microengineering (IMT), Photovoltaics and \\ Thin-Film Electronics Laboratory, Breguet 2, CH-2000 Neuchâtel, Switzerland
}

(Received 14 May 2013; accepted 1 August 2013; published online 15 August 2013)

\begin{abstract}
In amorphous/crystalline silicon heterojunction solar cells, an inversion layer is present at the front interface. By combining numerical simulations and experiments, we examine the contribution of the inversion layer to lateral transport and assess whether this layer can be exploited to replace the front transparent conductive oxide (TCO) in devices. For this, heterojunction solar cells of different areas $\left(2 \times 2,4 \times 4\right.$, and $\left.6 \times 6 \mathrm{~mm}^{2}\right)$ with and without TCO layers on the front side were prepared. Laser-beam-induced current measurements are compared with simulation results from the ASPIN2 semiconductor simulator. Current collection is constant across millimeter distances for cells with TCO; however, carriers traveling more than a few hundred microns in cells without TCO recombine before they can be collected. Simulations show that increasing the valence band offset increases the concentration of holes under the surface of n-type crystalline silicon, which increases the conductivity of the inversion layer. Unfortunately, this also impedes transport across the barrier to the emitter. We conclude that the lateral conductivity of the inversion layer may not suffice to fully replace the front TCO in heterojunction devices. (C) 2013 AIP Publishing LLC.
\end{abstract}

[http://dx.doi.org/10.1063/1.4818709]

\section{INTRODUCTION}

Hydrogenated amorphous silicon (a-Si:H)/crystalline silicon (c-Si) heterojunction solar cells are promising candidates for high-efficiency low-cost solar cells. With stacks of intrinsic and doped a-Si:H layers, excellent surface passivation can be achieved, leading to high open-circuit voltages in excess of $720 \mathrm{mV} .{ }^{1,2}$ The drawback of the amorphous emitter is its low mobility, which, together with a thickness limited to a few nanometers to reduce light absorption losses, ${ }^{3,4}$ results in a high resistivity. To overcome this disadvantage and achieve high efficiencies, a transparent conductive oxide (TCO) layer is required on top of the emitter for lateral transport of photogenerated charge carriers to metal finger contacts; the TCO also serves as a single-layer antireflection coating. To reduce optical losses associated with free-carrier absorption in TCOs, ${ }^{5}$ new high-mobility materials such as hydrogenated indium oxide are being introduced. ${ }^{6-8}$

Another feature of the a-Si:H emitter is that it forms a heterojunction with c-Si due to their different bandgaps and electron affinities. As a consequence of the band discontinuity at the heterojunction, band bending in $\mathrm{c}-\mathrm{Si}$ is increased compared to a homojunction and the hole concentration at the surface of the n-type c-Si exceeds the concentration of majority electrons in the bulk, thus forming an inversion layer (inset in Figure 5).

Recently, there has been experimental confirmation of the existence of the inversion layer using capacitive methods, ${ }^{9,10}$ planar conductivity measurements, ${ }^{11-13}$ and conductive probe atomic force microscopy. ${ }^{14,15}$ Until now, the

a)miha.filipic@fe.uni-lj.si inversion layer has been considered only as a tool to characterize the band offsets at the a-Si:H/c-Si interface ${ }^{16,17}$ and its influence on lateral transport in operating devices has not yet been analyzed. Lateral transport through an inversion layer is successfully leveraged in modulationdoped field-effect transistors $^{18}$ and in metal-insulatorconductor inversion layer solar cells. ${ }^{19-23}$ This prompted us to examine whether this layer can be used for lateral transport in heterojunction solar cells, with the specific aim of assessing TCO-free heterojunction solar cells. Such cells would feature an improved blue and red response, be potentially cheaper, and avoid passivation layer damage related to TCO sputtering, ${ }^{24}$ compared to our standard devices. ${ }^{25}$ When the TCO is absent, the a-Si:H emitter is too resistive to contribute noticeably to the lateral conductivity and has the same role as the insulator in metal-insulatorsemiconductor solar cells of inducing an inversion layer in c-Si. However, while in metal-insulator-semiconductor solar cells, the inversion layer is induced by fixed surface charges, the inversion layer in a-Si:H/c-Si solar cells is a consequence of the a-Si:H emitter doping and the band discontinuities at the interface, introducing new parameters to alter the conductivity of the inversion layer.

A schematic representation of the TCO-free concept is presented in Figure 1, highlighting the difference between devices with a TCO layer between the metal fingers (Figure 1(a)) and devices without such layers (Figure 1(b)). The hole mobility in the inversion layer is lower than in bulk c-Si due to carrier surface scattering, ${ }^{26}$ but it is still higher than in TCOs. Consequently, for the same specific resistance, a lower concentration of charge carriers in the channel is needed, reducing free-carrier absorption. 


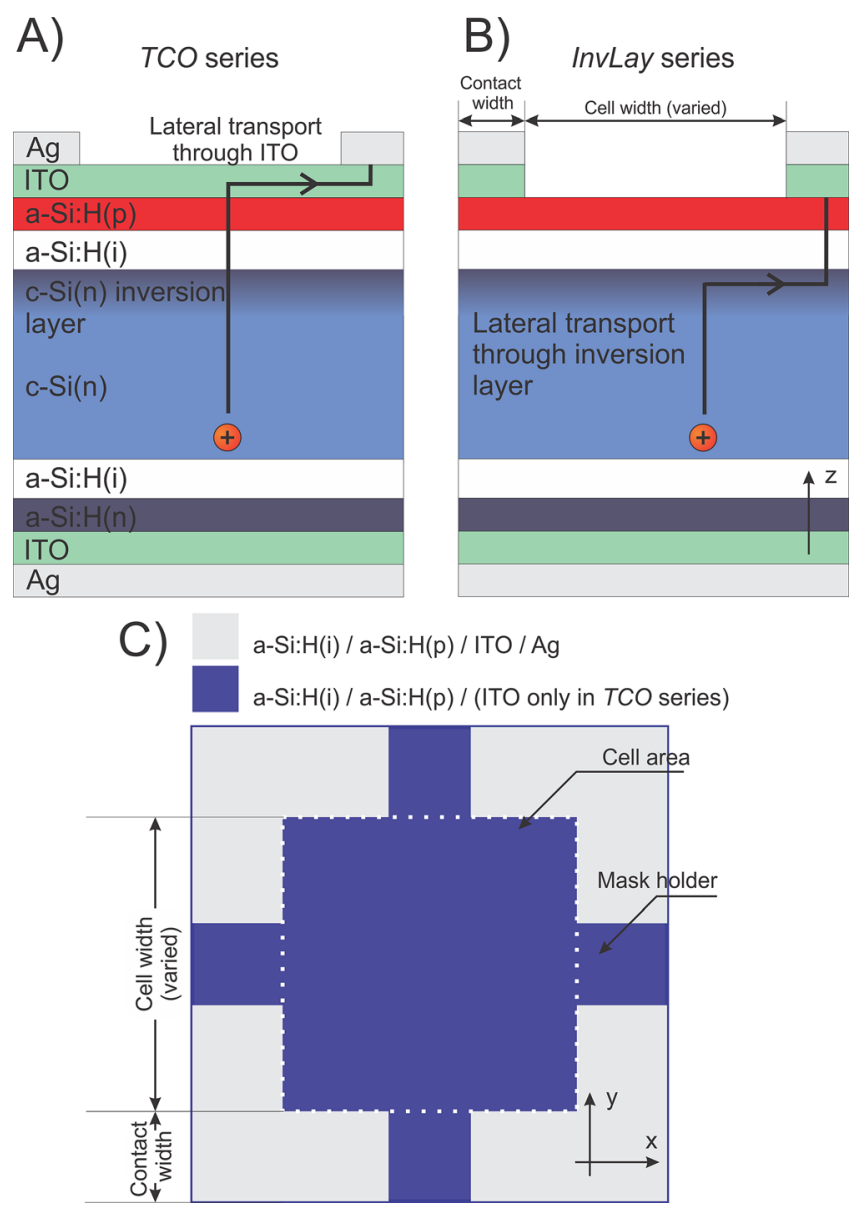

FIG. 1. (a) Schematic of a solar cell in the TCO series, with lateral transport of collected photogenerated holes through the ITO layer. (b) Schematic of a solar cell in the InvLay series, with lateral transport through the inversion layer. (c) Schematic of finished cells (top view).

In this paper, we employ numerical simulations as well as experiments to examine the contribution of the inversion layer to the lateral transport of photogenerated carriers on their way to the front external contact. Laser-beam-induced current (LBIC) maps of specially designed heterojunction solar cells with and without TCO layers of various areas were measured. ASPIN2, a two-dimensional solar cell simulator, ${ }^{27}$ was used to simulate one-dimensional LBIC profiles of these cells and evaluate how different parameters (i.e., band discontinuities) impact the lateral transport.

\section{EXPERIMENTAL AND SIMULATIONS}

As bases to make the cells, $3 \Omega \mathrm{cm}$, n-type, (100) polished, $280 \mu \mathrm{m}$-thick, float-zone wafers were used onto which intrinsic and doped a-Si:H layers were deposited by plasma-enhanced chemical vapor deposition (PECVD) ${ }^{28}$ Indium tin oxide (ITO) was sputtered $^{5}$ and silver was evaporated over the entire back side of each cell. Laser-cut silicon shadow masks were aligned by eye to cover the cell area during deposition of the front contact, defining cells with areas of $2 \times 2,4 \times 4$, and $6 \times 6 \mathrm{~mm}^{2}$, as shown in Figure 1(c). Two different series of cells were made: The "TCO" series (Figure 1(a)) had ITO sputtered over the entire front side and only silver evaporated through the mask, while the "InvLay" series (Figure 1(b)) had both ITO sputtered and silver evaporated through the mask. InvLay series cells thus do not have an ITO layer on the front of the active cell area. The ITO layer was present under the silver contacts in both cases to ensure goodquality electrical contacts. ${ }^{5}$ For the InvLay series, the masks were aligned twice, first for ITO sputtering and second for silver evaporation. Each series was co-deposited on the same wafer, which was cut in half before processing. Due to masking, the device structure has four front contacts, one in each corner, which were all electrically connected with contacting probes to form one front contact.

LBIC was measured by using an apparatus developed at the University of Ljubljana. ${ }^{29}$ A laser diode with a wavelength of $638 \mathrm{~nm}$ was used as a light source. A laser diode controller and waveform generator were used to drive the $49-\mathrm{Hz}$ sine waveform current through the diode. A beam splitter split the collimated beam in half; half the beam illuminated the measured cell and half illuminated a reference detector, which was used to compensate variations in the laser diode intensity. The beam illuminating the cell was passed through a focusing lens, giving a knife-edge beam width of $85 \mu \mathrm{m}$. The power of the beam illuminating the cell was $6.3 \mu \mathrm{W}$, as measured with a reference detector. This power was also used as the input beam power for LBIC simulations. The cell current was passed through a $10 \Omega$ resistor, which set the operating point sufficiently close to shortcircuit conditions. The amplitude of the voltage drop across the resistor was measured with a lock-in amplifier that responded only to the modulated laser beam. Linear stage controllers moved the measured cell in steps of $75 \mu \mathrm{m}$, giving a two-dimensional map of the cell's short-circuit current response.

ASPIN2, a numerical solar cell simulation software capable of simulating cell structures in two dimensions, was used to calculate one-dimensional LBIC profiles. The simulation structures are shown in Figures 1(a) and 1(b). To simulate surface states, a 1-nm-thick defect layer was introduced on both sides of the c-Si absorber with a volume defect density of $10^{14} \mathrm{~cm}^{-3}$, corresponding to a surface defect density of $10^{7} \mathrm{~cm}^{-2}$. With the data for c-Si from Table I, the surface defect density (neglecting the field-effect passivation) results in a surface recombination velocity of $1 \mathrm{~cm} / \mathrm{s}$ and an effective lifetime of $3.7 \mathrm{~ms}$, consistent with regularly measured lifetimes. $^{25}$

ITO was modeled as a conductor that contributed only to the series resistance; we neglected other effects like band bending and band-to-band tunneling. ${ }^{30}$ The thickness of the ITO was $65 \mathrm{~nm}$ and its mobility and free-carrier concentration are $30 \mathrm{~cm}^{2} /(\mathrm{Vs})$ and $3 \times 10^{20} \mathrm{~cm}^{-3}$, respectively, giving it a sheet resistance of $107 \Omega /$ sq. Generation rate profiles were calculated with the SunShine optical simulator, ${ }^{31}$ since more advanced features like ray tracing ${ }^{32}$ were not required for the polished wafers. Tunneling of holes through the a-Si:H/c-Si barrier was not included in the simulations. The input parameters used in the simulations are presented in Table I. Thicknesses of the layers were measured, while other parameters were taken from the literature. 
TABLE I. Simulation input parameters. The thermal velocity was $10^{7} \mathrm{~cm} / \mathrm{s}$ for all simulations.

\begin{tabular}{|c|c|c|c|c|c|}
\hline \multicolumn{2}{|l|}{ Parameter } & a-Si:H(p) & a-Si:H(i) & a-Si:H(n) & $\mathrm{c}-\mathrm{Si}$ \\
\hline \multicolumn{2}{|l|}{ Thickness (nm) } & 8 & 4 & 10 & 280000 \\
\hline \multicolumn{2}{|l|}{ Bandgap (eV) } & 1.7 & 1.7 & 1.7 & 1.124 \\
\hline \multicolumn{2}{|c|}{ Electron affinity $(\mathrm{eV})$} & 3.9 & 3.9 & 3.9 & 4.05 \\
\hline \multicolumn{2}{|c|}{ Acceptor/donor doping concentration $\left(\mathrm{cm}^{-3}\right)$} & $9 \times 10^{18} / 0$ & $0 / 0$ & $0 / 1 \times 10^{19}$ & $0 / 1.5 \times 10^{15}$ \\
\hline \multicolumn{2}{|c|}{ Electron/hole mobility $\left(\mathrm{cm}^{2} /(\mathrm{Vs})\right)$} & $10 / 2$ & $10 / 2$ & $10 / 2$ & $1300 / 450$ \\
\hline \multicolumn{2}{|c|}{ Effective DOS in CB/VB $\left(\mathrm{cm}^{-3}\right)$} & $2.00 \times 10^{20} / 2.00 \times 10^{20}$ & $2.00 \times 10^{20} / 2.00 \times 10^{20}$ & $2.00 \times 10^{20} / 2.00 \times 10^{20}$ & $2.80 \times 10^{19} / 2.65 \times 10^{19}$ \\
\hline \multicolumn{2}{|c|}{ Relative permittivity } & 11.9 & 11.9 & 11.9 & 11.9 \\
\hline \multirow[t]{3}{*}{$\mathrm{CB}$ tail states } & Total states $\left(\mathrm{cm}^{-3}\right)$ & $3 \times 10^{18}$ & $3 \times 10^{18}$ & $3 \times 10^{18}$ & $\ldots$ \\
\hline & Characteristic energy (eV) & 0.03 & 0.03 & 0.03 & $\ldots$ \\
\hline & e/h capture cross-section $\left(\mathrm{cm}^{-2}\right)$ & $10^{-16} / 10^{-15}$ & $10^{-16} / 10^{-15}$ & $10^{-16} / 10^{-15}$ & $\cdots$ \\
\hline \multirow[t]{3}{*}{ VB tail states } & Total states $\left(\mathrm{cm}^{-3}\right)$ & $5 \times 10^{18}$ & $5 \times 10^{18}$ & $5 \times 10^{18}$ & $\ldots$ \\
\hline & Characteristic energy $(\mathrm{eV})$ & 0.05 & 0.05 & 0.05 & $\ldots$ \\
\hline & $\mathrm{e} / \mathrm{h}$ capture cross-section $\left(\mathrm{cm}^{-2}\right)$ & $10^{-15} / 10^{-16}$ & $10^{-15} / 10^{-16}$ & $10^{-15} / 10^{-16}$ & $\ldots$ \\
\hline \multirow{6}{*}{$\begin{array}{l}\text { Dangling bond } \\
\text { states }\end{array}$} & Total states $\left(\mathrm{cm}^{-3}\right)$ & $10^{18}$ & $10^{16}$ & $10^{18}$ & $\ldots$ \\
\hline & $\mathrm{E}_{+/ 0} / \mathrm{E}_{0 /-}(\mathrm{eV})$ & $0.8 / 1$ & $0.7 / 0.9$ & $0.5 / 0.7$ & $\ldots$ \\
\hline & $\sigma(\mathrm{eV})$ & 0.22 & 0.22 & 0.22 & $\ldots$ \\
\hline & $\begin{array}{l}\text { e/h neutral capture cross-section } \\
\left(\mathrm{cm}^{-2}\right)\end{array}$ & $10^{-14} / 10^{-14}$ & $10^{-14} / 10^{-14}$ & $10^{-14} / 10^{-14}$ & $\ldots$ \\
\hline & $\begin{array}{l}\text { e/h charged capture cross-section } \\
\left(\mathrm{cm}^{-2}\right)\end{array}$ & $10^{-15} / 10^{-15}$ & $10^{-15} / 10^{-15}$ & $10^{-15} / 10^{-15}$ & $\ldots$ \\
\hline & Correlation energy (eV) & 0.2 & 0.2 & 0.2 & $\ldots$ \\
\hline \multirow{2}{*}{$\begin{array}{l}\text { Midgap states } \\
\text { e/h capture } \\
\text { cross-section } \\
\left(\mathrm{cm}^{-2}\right)\end{array}$} & Total states $\left(\mathrm{cm}^{-3}\right)$ & $\ldots$ & $\ldots$ & $\ldots$ & $2 \times 10^{9}$ \\
\hline & $\ldots$ & $\ldots$ & $\ldots$ & $10^{-14} / 10^{-14}$ & \\
\hline
\end{tabular}

\section{RESULTS AND DISCUSSION}

First, we compare the free-carrier absorption of an optimized front ITO layer ${ }^{3,5}$ and an equivalent-resistance c-Si inversion layer to evaluate the potential short-circuit current gain in a TCO-free heterojunction solar cell. We chose a wavelength of $1000 \mathrm{~nm}$ where $\mathrm{c}-\mathrm{Si}$ is still absorbing and free-carrier absorption begins to occur. At this wavelength, the absorption coefficient of our ITO, measured by ellipsometry, ${ }^{5}$ is $5000 \mathrm{~cm}^{-1}$. To get an estimate of the inversion layer absorption coefficient, we follow Schroder ${ }^{33}$

$$
\alpha_{\mathrm{FCA}}=\frac{q^{3} \lambda^{2} p}{4 \pi^{2} \epsilon_{0} c^{3} n_{r} m^{* 2} \mu},
$$

where $\lambda$ is wavelength, $p$ is hole concentration, $n_{r}$ is refractive index, $m^{*}$ is effective mass, $\mu$ is mobility, and other constants have their usual meanings. To compare the absorption coefficients, we assume that the inversion layer has the same thickness and sheet resistance as the ITO $(65 \mathrm{~nm}$ and $107 \Omega / \mathrm{sq}$, respectively). Such a low sheet resistance of the inversion layer is unlikely to be obtained in practice (see Figures 5 and 6 and the corresponding discussion), and serves here only for the comparison of free-carrier absorption in the inversion layer and ITO. Supposing a low hole mobility at the surface of $\mathrm{c}-\mathrm{Si}$ of $150 \mathrm{~cm}^{2} /(\mathrm{Vs})$, we get a hole concentration of $6 \times 10^{19} \mathrm{~cm}^{-3}$, which together with an effective mass of $0.37 \times m_{0}$ and refractive index of 3.57 gives a c-Si inversion layer absorption coefficient of $64 \mathrm{~cm}^{-1}, 78$ times lower than that of the ITO. Based on previous experimental results in which the free-carrier density of the front ITO layer was varied in silicon heterojunction solar cells, ${ }^{5}$ we estimate that this would correspond to a gain of more than $0.5 \mathrm{~mA} / \mathrm{cm}^{2}$.

An LBIC scan measured in the dark is shown in Figure 2 for the $6 \times 6 \mathrm{~cm}^{2}$ TCO cell. Smaller area cells are not shown, since they have similar LBIC response. Cells in the TCO series show almost no variation in LBIC response over the entire area, as expected, indicating that the conductivity of the ITO layers is high enough not to hinder lateral

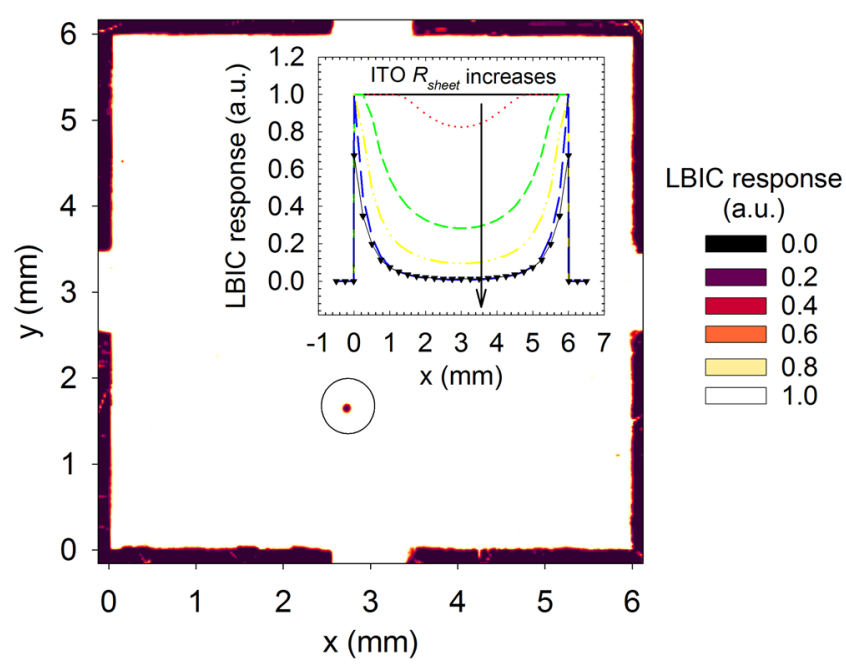

FIG. 2. LBIC scan of the $6 \times 6 \mathrm{~cm}^{2}$ TCO cell that has full ITO coverage. The circle marks a defect. The inset graph shows simulated one-dimensional LBIC profiles when increasing the ITO sheet resistance (values are 100, $300,1 \mathrm{k}, 3 \mathrm{k}$, and $1 \mathrm{M} \Omega / \mathrm{sq})$. The lowest line with symbols shows the simulated profile without an ITO layer. 
photogenerated carrier collection. Increasing the sheet resistance from $100 \Omega / \mathrm{sq}$ to $3 \mathrm{k} \Omega / \mathrm{sq}$ results in a drop of the LBIC profiles, as shown in the inset of Figure 2. For a very high resistance value of $1 \mathrm{M} \Omega / \mathrm{sq}$, the profile approaches the simulation profile without an ITO layer (line with symbols in the inset). Note that the sheet resistance of the doped a-Si:H emitter layer is $400 \mathrm{k} \Omega / \mathrm{sq}$. The difference close to the contacts is a consequence of the antireflection provided by the ITO.

An LBIC scan measured in the dark is shown in Figure 3 for the InvLay series. Cells in the InvLay series have high LBIC response only near the contacts, indicating that lateral transport is poor without the ITO layer. The inversion layer is apparently insufficiently conductive to transport holes across millimeter distances without significant recombination. Inspection of the cells under a microscope shows that the higher response along the top and left edges is a consequence of imperfect alignment of the deposition masks, as some ITO was not covered by evaporated silver (arrows in Figure 3).

Analysis of the TCO series shows that the effective defect area revealed in the LBIC scans is on average smaller than for the InvLay series. The exact origin of these anomalies during cell fabrication is not known. Some of the anomalies are marked with circles in Figures 2 and 3. The ITO layer reduces the area of an imperfection's influence, so its role is not only to provide lateral transport but also to facilitate collection of carriers near the defective regions.

Measured one-dimensional LBIC profiles in the dark are compared with simulated values in Figure 4. For each twodimensional LBIC scan, one vertical and one horizontal profile were chosen. The vertical profiles were chosen closer to the middle of the cell than the horizontal profiles, as indicated by the white dashed lines in Figure 3, showing how the distance from the contacts perpendicular to a profile affects that profile. LBIC profiles are centered at the middle of the cell for a clearer plot. The measured profiles reach an asymmetric maximum because of the misalignment of the mask. The simulated profiles for the smaller $2 \times 2$ and $4 \times 4 \mathrm{~mm}^{2}$ cells show deeper drops in response at the center of the cells

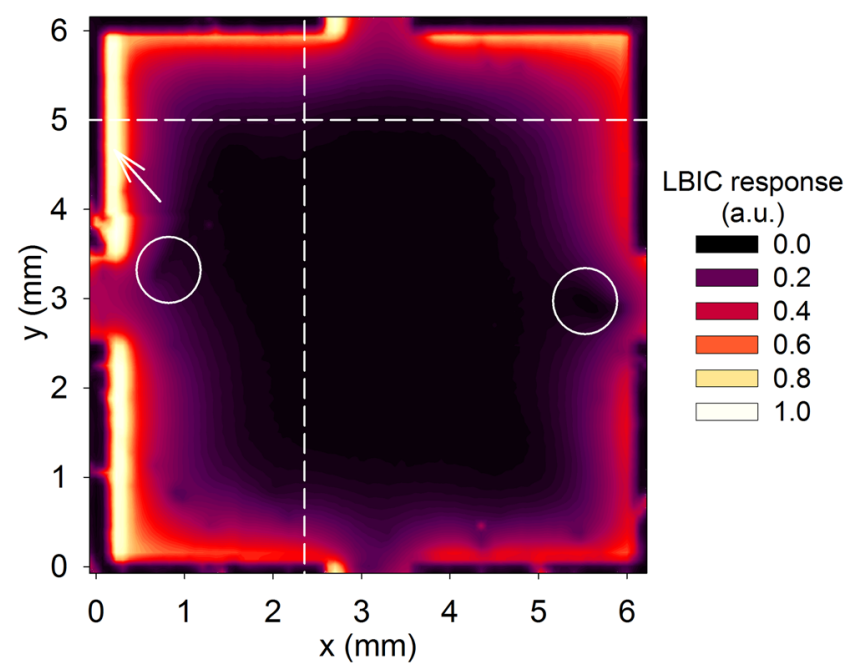

FIG. 3. LBIC scan of the $6 \times 6 \mathrm{~cm}^{2}$ InvLay cell, which has no ITO on the active cell area. Dashed lines show the location of the widest $6 \mathrm{~cm}$ profile in Figure 4. Arrows point to areas covered with ITO but not silver due to slight misalignment of the mask. Circles mark defects.

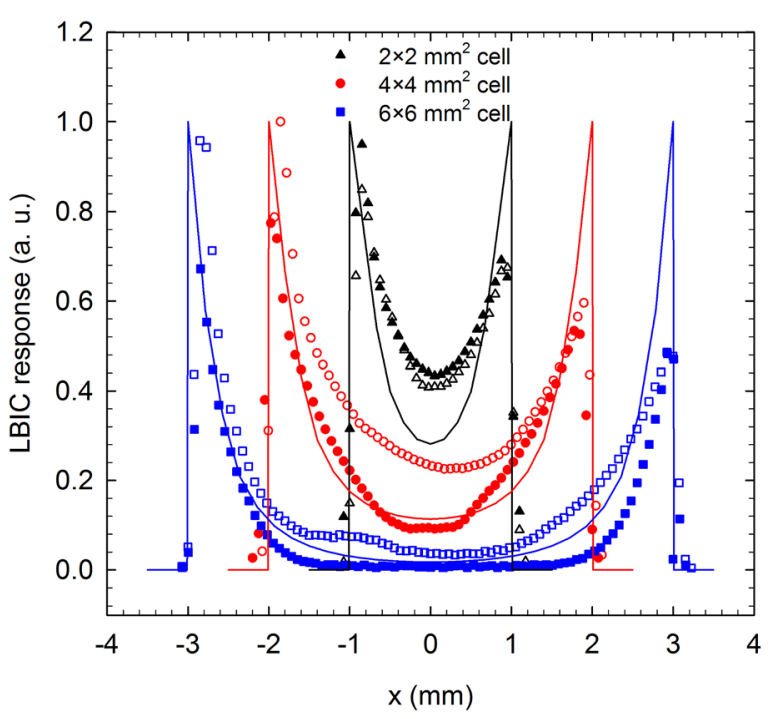

FIG. 4. Measured and simulated LBIC profiles. Full symbols and empty symbols are the vertical and horizontal profiles marked by dashed lines in Figure 3, respectively. Lines represent simulated LBIC profiles.

compared to the measurements, since in the two-dimensional simulation, contacts are only on the sides and not all around the cell like in the measurements. Nevertheless, the simulations faithfully reproduce the shape and approximate values of the measured LBIC profiles, and thus allow us to explore the effect on inversion layer transport of changing parameters that are at present difficult to control experimentally.

The simulated and measured LBIC response at the middle of the cells is unacceptably low (if the inversion layer is to replace the front TCO), but rises with decreasing cell area. To extract more current from the InvLay series of cells, the contacts have to be closer together or the conductivity of the inversion layer needs to be increased. Simulations indicate that for the LBIC profile to drop by less than $5 \%$ of its maximum value the contact spacing should be less than $0.75 \mathrm{~mm}$. Assuming a metal finger width of $75 \mu \mathrm{m}$, almost $10 \%$ of the cell area

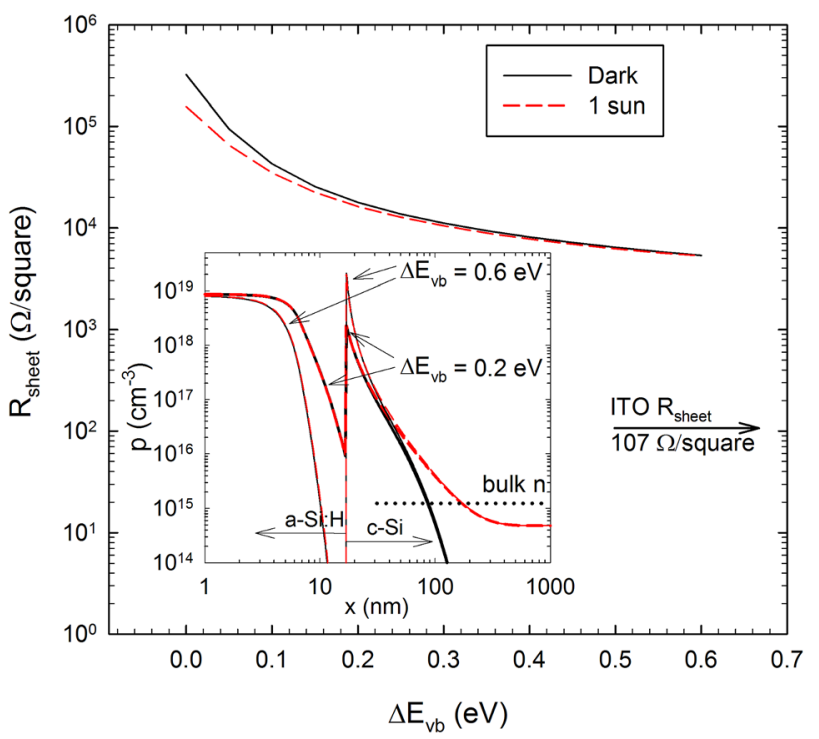

FIG. 5. Inversion layer sheet resistance dependence on $\Delta E_{v b}$. Inset shows the hole concentration profile near the junction (between the contacts) for two different $\Delta E_{v b}$. 

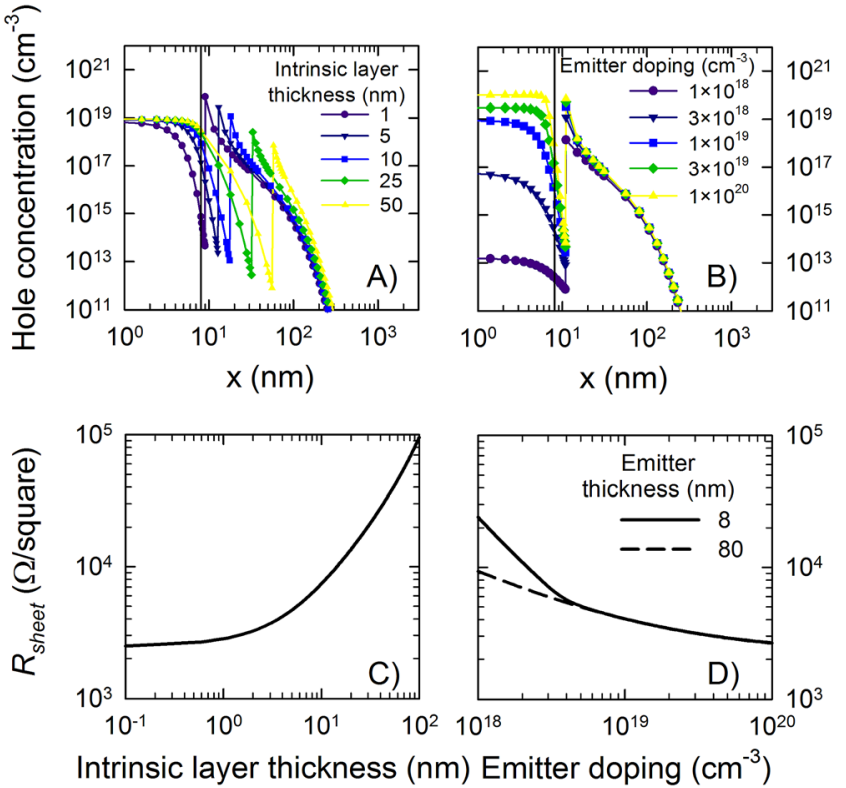

FIG. 6. Hole concentration profiles for several intrinsic layer thicknesses (a) and emitter doping concentrations (b). The black vertical lines indicate the interface of the doped and intrinsic a-Si:H layers. The c-Si starts at the discontinuous jump in hole concentration. Inversion layer sheet resistance as a function of intrinsic layer thickness (c) and emitter doping concentration (d).

would be shaded by the metallic front grid. This leaves inversion layer conductivity increase as the only viable option for lateral transport through the inversion layer.

In order to increase the conductivity of the inversion layer, more carriers need to be present at the surface of the c-Si absorber. This can be achieved for n-type c-Si by a higher valence band offset $\left(\Delta E_{v b}\right)$ at the a-Si:H/c-Si heterojunction, which increases the barrier height but also increases the band bending and hole concentration in c-Si. The driving force due to increased $\Delta E_{v b}$, which pulls holes from the p-type a-Si:H emitter into the inversion layer, acting against the built-in electric field, is not sufficient to create a highly conductive inversion layer. To effectively increase the hole concentration in the inversion layer, both a large enough driving force and a suitably large amount of holes in the p-type a-Si:H emitter are needed, demanding a high $\Delta E_{v b}$ and a sufficiently doped and thick emitter. ${ }^{18}$ In practice, the $\Delta E_{v b}$ can be increased by $0.15 \mathrm{eV}$ by increasing the a-Si:H hydrogen content from $12 \%$ to $24 \% .{ }^{34}$ Another possibility is to deposit hydrogenated amorphous silicon suboxide films $\left(\mathrm{a}-\mathrm{SiO}_{\mathrm{x}}: \mathrm{H}\right)$ with higher bandgaps than a-Si:H. ${ }^{35,36}$ In the simulations, the initial $\Delta E_{v b}$ of $0.43 \mathrm{eV}$ was varied by hypothetically changing the front a-Si:H layers' bandgap, while leaving all other parameters the same. Note that widening the bandgap of the emitter and front intrinsic a-Si:H layers would also decrease the UV and visible parasitic absorption in these layers. ${ }^{3}$

The simulated sheet resistance of the inversion layer is shown in Figure 5. The sheet resistance was calculated according to the following equation:

$$
R_{s h}=\frac{1}{q \mu_{p} \int_{0}^{d_{i n v}} p \cdot d x},
$$

where $q$ is the elementary charge, $\mu_{p}$ is the bulk hole mobility taken from Table I, $p$ is the hole concentration, and $d_{i n v}$ is the thickness of the inversion layer. The hole concentration was averaged from the surface of $\mathrm{c}-\mathrm{Si}$ to $d_{i n v}$, which is defined as the thickness at which the hole concentration drops below the majority electron concentration in the bulk c-Si (marked by the dotted line in the inset of Figure 5). By integrating only to $d_{i n v}$, the contribution of photogenerated holes in bulk c-Si is removed. Increasing $\Delta E_{v b}$ at the a-Si:H/ $\mathrm{c}-\mathrm{Si}$ interface increases the hole concentration at the surface of the c-Si, as can be seen in the inset of Figure 5. Favre et $a l .{ }^{13}$ simulated the same structure without the intrinsic layer, which changes the band bending, but at room temperature our values of the surface hole concentration, obtained by evaluating the integral in Eq. (2) without illumination, have the same $\Delta E_{v b}$ trend. The dashed line shows the sheet resistance at 1-sun illumination, which is only slightly lower than in the dark. Thus, although our LBIC measurements were performed in the dark, the inversion layer will not become significantly more conductive when illuminated, as in an operating device. From the inset, it can be seen that when illumination is applied the peak concentration of holes at the c-Si surface increases negligibly, but the concentration at a depth of $100 \mathrm{~nm}$ increases by nearly an order of magnitude, which also increases $d_{i n v}$. The illumination influence on holes in the a-Si:H layers is negligible. Note that the actual hole mobility in the inversion layer is lower than the assumed bulk value, ${ }^{26}$ which additionally increases the inversion layer sheet resistance. Even in the best case, with $\Delta E_{v b}=0.6 \mathrm{eV}$ and the mobility value taken from the bulk, the sheet resistance of the inversion layer is two orders of magnitude higher than the ITO sheet resistance.

The concentration of holes in the c-Si inversion layer is also affected by the thickness of the intrinsic layer and the doping concentration of the emitter. The plots in Figure 6 show how the intrinsic layer thickness and the emitter doping concentration influence the hole concentration profile and consequently the sheet resistance calculated according to Eq. (2). Since illumination only slightly alters the inversion layer sheet resistance, these simulations were done in the dark with data from Table I $\left(\Delta E_{v b}=0.43 \mathrm{eV}\right)$. The density of surface defect states was kept constant, regardless of the intrinsic layer thickness. The intrinsic a-Si:H layer provides no additional free holes and increasing its thickness results in a lower hole concentration in the c-Si (Figure 6(a)). The peak hole concentration in the inversion layer decreases and moves further away from the doped a-Si:H layer. The sheet resistance of the inversion layer starts to significantly increase for intrinsic layer thicknesses greater than $3 \mathrm{~nm}$ (Figure 6(c)). For a constant intrinsic layer thickness of $4 \mathrm{~nm}$, the depletion region in the emitter is narrower than the emitter for high doping concentration (above $3 \times 10^{18} \mathrm{~cm}^{-3}$ for an 8-nm-thick emitter). In this case, lowering the doping concentration slightly reduces the hole concentration in the c-Si inversion layer (Figure 6(b)). Once the doping is low enough that the emitter depletion region extends across its entire thickness, the hole concentration is drastically lowered in the emitter as well as in the c-Si inversion layer. The sheet resistance of the inversion layer then exhibits a sharp 
increase with decreasing doping concentration (Figure 6(d), full line). As long as the emitter is thicker than the depletion region, this sharp increase of sheet resistance is absent (Figure 6(c) dashed line). Even for the thinnest intrinsic layer and the highest emitter doping concentration, the sheet resistance of the inversion layer is still more than an order of magnitude higher than the sheet resistance of the ITO. As with varying $\Delta E_{v b}$, thinning the intrinsic layer or increasing the doping of the emitter is insufficient to achieve a low inversion layer sheet resistance.

In addition to altering the inversion layer, changing the band offset or intrinsic layer thickness influences the hole collection. The effect of $\Delta E_{v b}$ on LBIC response was simulated and the results are shown in Figure 7 for the InvLay $2 \times 2 \mathrm{~mm}^{2}$ cell. With increasing $\Delta E_{v b}$ from 0 to $0.5 \mathrm{eV}$, the LBIC profiles rise because of the decrease in the inversion layer sheet resistance, which enhances collection of photogenerated carriers from the middle of the cell. The LBIC profile responds similarly if the inversion layer sheet resistance is reduced by a thinner intrinsic layer or higher-doped emitter. After the offset exceeds $0.5 \mathrm{eV}$, however, a sharp decrease can be observed in both LBIC response and shortcircuit current (inset in Figure 7). In this case, $\Delta E_{v b}$ is high enough that thermionic emission over the barrier becomes the limiting transport mechanism, impeding transport of holes across the barrier to the emitter region.

To circumvent this problem, a patterned structure was simulated in which the bandgap of the a-Si:H layers was kept constant under the metal contacts to allow for good transverse transport. Here, $\Delta E_{v b}$ was varied only under the active cell area. This structure could be made in practice by using shadow masks during PECVD depositions. With this structure, the barrier height under the contacts is kept low enough that thermionic emission does not become the limiting mechanism and hole collection is not impeded. LBIC simulations in Figure 8, and the short-circuit current in the inset, show an increase in collection with higher valence band offset, until

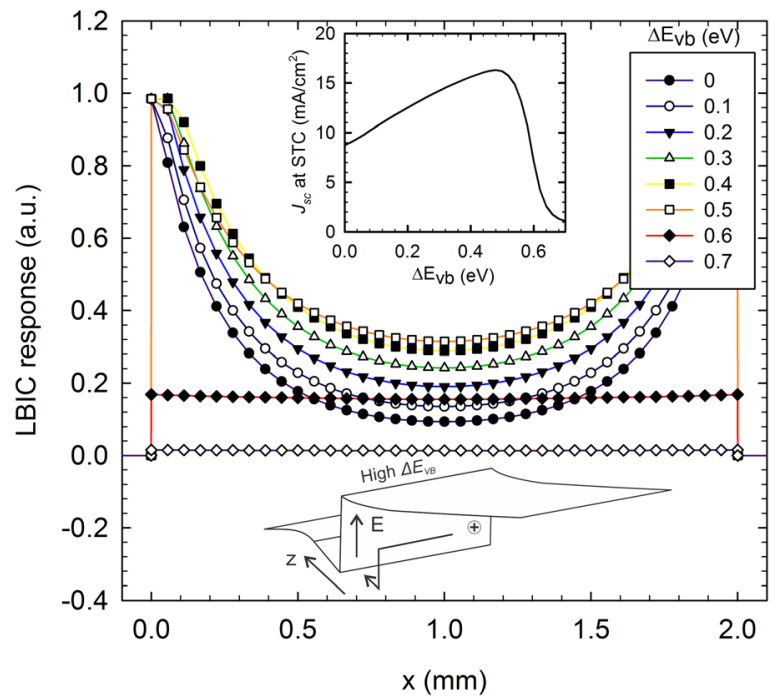

FIG. 7. Simulated effect of $\Delta E_{v b}$ on the dark LBIC profiles of the $2 \times 2 \mathrm{~mm}^{2}$ InvLay cell. The inset shows the short-circuit current at 1 sun illumination. The schematic at the bottom illustrates lateral hole transport and thermionic emission over the barrier.

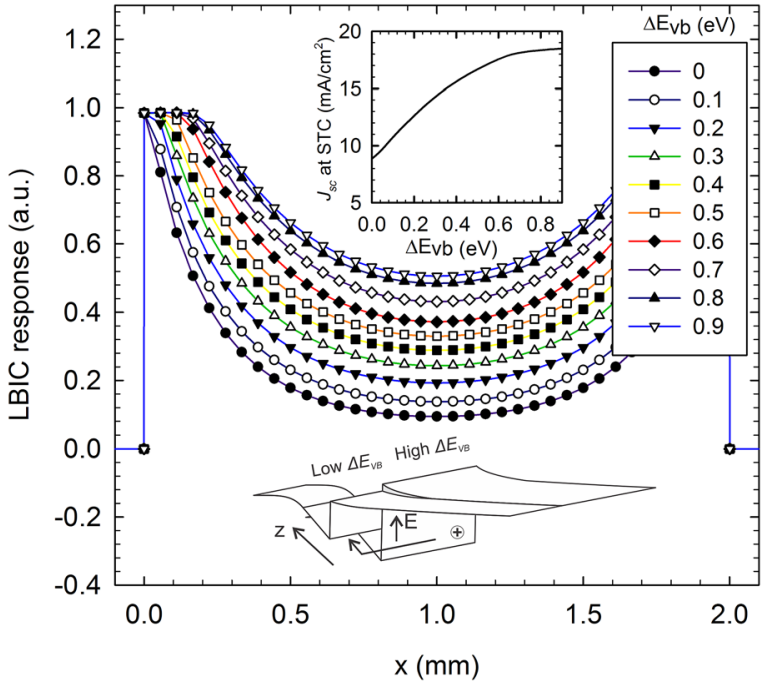

FIG. 8. Simulated effect of $\Delta E_{v b}$ on the dark LBIC profiles of the $2 \times 2 \mathrm{~mm}^{2}$ InvLay cell for a patterned structure. The inset shows the short-circuit current at 1 sun illumination. The schematic at the bottom illustrates lateral hole transport and unobstructed collection under the contacts, where the barrier is lower.

saturation is reached. The Fermi level at the c-Si surface is in this case positioned very close to the valence band, and a further increase in charge concentration at the surface would require much higher band offsets as well as higher doping of the a-Si:H emitter. Even when this saturation occurs, the LBIC profile is not flat, as with an ITO layer.

Without patterning, the barrier height must be kept low enough not to inhibit hole collection under the contacts. The conductivity of the inversion layer cannot be varied much in this case and it, therefore, cannot replace the TCO layer. With patterning, the sheet resistance of the inversion layer can be decreased under the non-contacted area with more freedom. Nonetheless, Figure 8 reveals that even with patterned contacts and a large valence band offset of $0.9 \mathrm{eV}$, holes traveling more than approximately $200 \mu \mathrm{m}$ are prone to recombine before collection. Simulation results indicate that to achieve a flat LBIC profile for a $2 \times 2 \mathrm{~mm}^{2}$ cell, the acceptor doping in the a-Si:H(p) layer would have to be increased tenfold and the valence band offset would have to be higher than $2.6 \mathrm{eV}$, leading to strong inversion in the c-Si. Such high offsets cannot be achieved in practice with $\mathrm{a}-\mathrm{Si}: \mathrm{H},{ }^{34}$ but might be possible with other materials.

Other approaches are needed if lateral transport is to be conducted through the inversion layer. One possible improvement is to use p-type wafers. Electrons in c-Si have approximately three times higher mobility than holes, which would decrease the sheet resistance of the inversion layer. Another possibility to create the inversion layer under the active cell area is to use the metal-insulator-semiconductor approach with novel organic materials. ${ }^{37}$

\section{CONCLUSION}

The contribution of the inversion layer to lateral transport of photogenerated holes was studied with numerical simulations and LBIC measurements. Heterojunction solar cells of different area $\left(2 \times 2,4 \times 4\right.$, and $\left.6 \times 6 \mathrm{~mm}^{2}\right)$ with and 
without front ITO layers were prepared. For the TCO series of cells, no variation with position is observed. Increasing $\Delta E_{v b}$ at the a-Si:H/c-Si interface increases the band bending in $\mathrm{c}-\mathrm{Si}$, resulting in an increased hole concentration under the surface of the c-Si. The increased concentration leads to a lower sheet resistance of the inversion layer, which facilitates photogenerated charge carrier collection from the middle of the cell. Based on the simulation results, the required low sheet resistance of the inversion layer cannot be achieved with the a-Si:H materials system, and the inversion layer cannot replace the ITO layer for a high-efficiency cell on an n-type c-Si substrate.

\section{ACKNOWLEDGMENTS}

The authors would like to thank Antoine Descoeudres, Johannes Peter Seif, and Loris Barraud for their help in making samples, and Marko Berginc for help with measurements. Funding for this work was provided by the European Union Seventh Framework Programme (FP7/2007-2013), Collaborative Project (CP) "20pl $\mu$ s," Axpo Naturstrom Fonds, Switzerland, the Swiss CTI (Project 9908 PFNMMNM) The Slovenian Research Agency (P2-0197), and The Slovene Human Resources and Scholarship Fund.

${ }^{1}$ S. De Wolf, A. Descoeudres, Z. C. Holman, and C. Ballif, Green 2, 7 (2012).

${ }^{2}$ T. Mishima, M. Taguchi, H. Sakata, and E. Maruyama, Sol. Energy Mater. Sol. Cells 95, 18 (2011).

${ }^{3}$ Z. C. Holman, A. Descoeudres, L. Barraud, F. Z. Fernandez, J. P. Seif, S. De Wolf, and C. Ballif, IEEE J. Photovoltaics 2, 7 (2012).

${ }^{4}$ M. Tanaka, M. Taguchi, T. Matsuyama, T. Sawada, S. Tsuda, S. Nakano, H. Hanafusa, and Y. Kuwano, Jpn. J. Appl. Phys., Part 1 31, 3518 (1992).

${ }^{5}$ Z. C. Holman, M. Filipič, A. Descoeudres, S. De Wolf, F. Smole, M. Topič, and C. Ballif, J. Appl. Phys. 113, 013107 (2013).

${ }^{6}$ T. Koida, H. Fujiwara, and M. Kondo, Jpn. J. Appl. Phys., Part 2 46, L685 (2007).

${ }^{7}$ L. Barraud, Z. C. Holman, N. Badel, P. Reiss, A. Descoeudres, C. Battaglia, S. De Wolf, and C. Ballif, Sol. Energy Mater. Sol. Cells 115, 151 (2013).

${ }^{8}$ T. Koida, H. Fujiwara, and M. Kondo, Appl. Phys. Express 1, 041501 (2008).

${ }^{9}$ A. S. Gudovskikh, S. Ibrahim, J.-P. Kleider, J. Damon-Lacoste, P. Roca i Cabarrocas, Y. Veschetti, and P.-J. Ribeyron, Thin Solid Films 515, 7481 (2007).
${ }^{10}$ J. V. Li, R. S. Crandall, D. L. Young, M. R. Page, E. Iwaniczko, and Q. Wang, J. Appl. Phys. 110, 114502 (2011).

${ }^{11}$ J.-P. Kleider, Y. M. Soro, R. Chouffot, A. S. Gudovskikh, P. Roca i Cabarrocas, J. Damon-Lacoste, D. Eon, and P.-J. Ribeyron, J. Non-Cryst. Solids 354, 2641 (2008).

${ }^{12}$ J.-P. Kleider, A. S. Gudovskikh, and P. Roca i Cabarrocas, Appl. Phys. Lett. 92, 162101 (2008).

${ }^{13}$ W. Favre, M. Labrune, F. Dadouche, A. S. Gudovskikh, P. Roca i Cabarrocas, and J.-P. Kleider, Phys. Status Solidi C 7, 1037-1040 (2010).

${ }^{14}$ J.-P. Kleider, J. Alvarez, A. V. Ankudinov, A. S. Gudovskikh, E. V. Gushchina, M. Labrune, O. A. Maslova, W. Favre, M.-E. Gueunier-Farret, P. Roca i Cabarrocas, and E. I. Terukov, Nanoscale Res. Lett. 6, 152 (2011).

${ }^{15}$ O. A. Maslova, J. Alvarez, E. V. Gushina, W. Favre, M. E. GueunierFarret, A. S. Gudovskikh, A. V. Ankudinov, E. I. Terukov, and J.-P. Kleider, Appl. Phys. Lett. 97, 252110 (2010).

${ }^{16}$ R. Varache, W. Favre, L. Korte, and J.-P. Kleider, J. Non-Cryst. Solids 358, 2236 (2012).

${ }^{17}$ R. Varache, J. P. Kleider, W. Favre, and L. Korte, J. Appl. Phys. 112, 123717 (2012).

${ }^{18}$ L. D. Nguyen, L. E. Larson, and U. K. Mishra, Proc. IEEE 80, 494 (1992).

${ }^{19}$ B. Kuhlmann, A. G. Aberle, R. Hezel, and G. Heiser, IEEE Trans. Electron Devices 47, 2167 (2000).

${ }^{20}$ A. G. Aberle, B. Kuhlmann, R. Meyer, A. Hübner, C. Hampe, and R. Hezel, Prog. Photovoltaics 4, 193 (1996).

${ }^{21}$ R. Hezel, R. Meyer, and A. Metz, Sol. Energy Mater. Sol. Cells 65, 311 (2001).

${ }^{22}$ Ş. Altındal, A. Tataroğlu, and İ. Dökme, Sol. Energy Mater. Sol. Cells 85, 345 (2005).

${ }^{23}$ M. A. Green and A. W. Blakers, Sol. Cells 8, 3 (1983).

${ }^{24}$ B. Demaurex, S. De Wolf, A. Descoeudres, Z. Charles Holman, and C. Ballif, Appl. Phys. Lett. 101, 171604 (2012).

${ }^{25}$ A. Descoeudres, Z. C. Holman, L. Barraud, S. Morel, S. De Wolf, and C. Ballif, IEEE J. Photovoltaics 3, 83 (2013).

${ }^{26}$ F. Fang and S. Triebwasser, IBM J. Res. Dev. 8, 410 (1964).

${ }^{27}$ M. Nerat, F. Smole, and M. Topič, Thin Solid Films 519, 7497 (2011).

${ }^{28}$ A. Descoeudres, L. Barraud, S. De Wolf, B. Strahm, D. Lachenal, C. Guérin, Z. C. Holman, F. Zicarelli, B. Demaurex, J. Seif, J. Holovsky, and C. Ballif, Appl. Phys. Lett. 99, 123506 (2011).

${ }^{29}$ M. Bokalič and M. Topič, in Proceedings of the 46th International Conference on Microelectronics, Devices and Materials (2010), p. 233.

${ }^{30}$ A. Kanevce and W. K. Metzger, J. Appl. Phys. 105, 094507 (2009).

${ }^{31}$ J. Krč, F. Smole, and M. Topič, Prog. Photovoltaics 11, 15 (2003).

${ }^{32}$ B. Lipovšek, J. Krč, and M. Topič, J. Microelectron. Electron. Compon. Mater. 41, 264 (2011).

${ }^{33}$ D. Schroder, R. N. Thomas, and J. C. Swartz, IEEE J. Solid-State Circuits 13, 180 (1978).

${ }^{34}$ T. F. Schulze, L. Korte, F. Ruske, and B. Rech, Phys. Rev. B 83, 165314 (2011).

${ }^{35}$ T. Mueller, J. Wong, and A. G. Aberle, Energy Proc. 15, 97 (2012).

${ }^{36}$ C. Banerjee, J. Sritharathikhun, A. Yamada, and M. Konagai, J. Phys. D: Appl. Phys. 41, 185107 (2008).

${ }^{37}$ A. S. Erickson, N. K. Kedem, A. E. Haj-Yahia, and D. Cahen, Appl. Phys. Lett. 101, 233901 (2012). 